\title{
Invasive Salmonellosis in a 45-Day-Old Infant
}

Joshua Klepinger, MD

\section{A previously healthy infant was presented for evaluation of persistent fever and fussiness.}

T he management of a febrile infant is complex and requires obtaining a detailed history of all possible exposures. Published guidelines alone are not always completely accurate for diagnosing or excluding serious illness, and are not a substitute for a thorough examination and history.

\section{Case}

The parents of a 45-day-old girl were referred to our regional pediatric hospital by a local community hospital for emergent evaluation of their infant. The day prior, they had taken the infant to the referring ED because of persistent fussiness and subjective fever. They were neither sure of the tests that were obtained during that visit nor why they were instructed to take their daughter to our pediatric facility. They did, however, recall that during the visit to the community ED, the patient had a rectal temperature of $102.7^{\circ} \mathrm{F}$, was given an antibiotic injection, and was well appearing and acting normally. Also, at discharge, the infant's parents were instructed to follow up with the patient's pediatrician within 24 hours.

Since their daughter's discharge from the community ED, both parents noted that she seemed more irritable, felt warm, and had not been feeding well. They confirmed that she was an otherwise healthy infant who had been born full term via normal vaginal delivery and without complications.

On initial assessment at our ED, the patient was fussy and had mottled extremities and dusky nail beds. Her vital signs at presentation were: heart rate, 223 beats/minute; respiratory rate, 36 breaths/ minute; and rectal temperature, $103.6^{\circ} \mathrm{F}$. Oxygen saturation was $96 \%$ on room air. The infant was resuscitated with $20 \mathrm{~mL} / \mathrm{kg}$ of intravenous (IV) normal saline and given oral acetaminophen. Laboratory studies were obtained, and a lumbar puncture (LP)

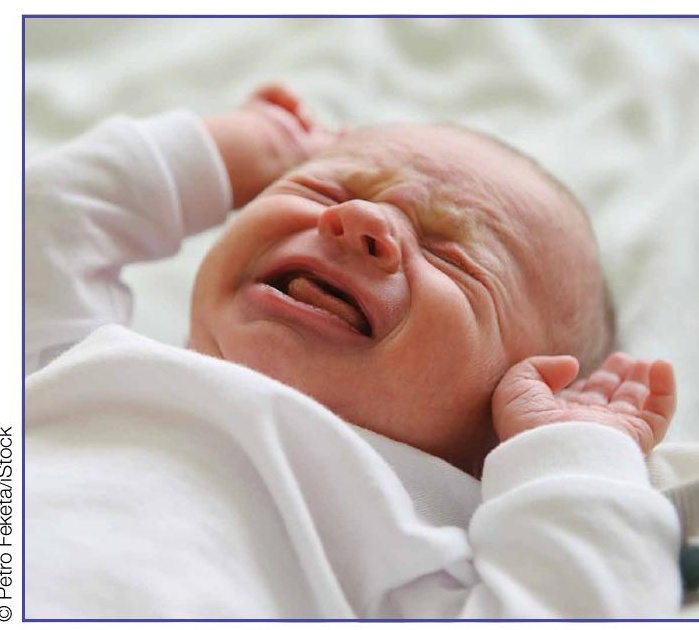
was performed.

She was treated with IV acyclovir, ceftriaxone, and vancomycin. Her complete blood count (CBC) was notable for a white blood cell count (WBC) of $22.60 \times 10^{9} / \mathrm{L}$; cerebrospinal fluid (CSF) analysis revealed a WBC of $4.52 \times 10^{9} / \mathrm{L}$ with $75 \%$ neutrophils, and serum glucose of $63 \mathrm{mg} / \mathrm{dL}$.

Since the patient's parents did not have any paperwork or information from the prior ED visit, our ED contacted the com- 
munity ED by phone, and a representative provided the following information: the patient had appeared well but was febrile at presentation; laboratory evaluation was obtained, but no LP was performed; she was treated with intramuscular (IM) cef-

Twenty-five percent of Salmonella infections in children younger than age 5 years have been attributed to contact with a pet.... triaxone and acetaminophen; and she was discharged home in the care of her parents. Regarding laboratory studies performed at the community ED, the only test result made available by phone was the preliminary blood culture report that revealed growth of gram-negative rods with speciation pending, which prompted the referral to our facility.

Based on the information provided by the community ED and our evaluation and work-up, the patient was admitted to the pediatric intensive care unit. Magnetic resonance imaging (MRI) of the brain was performed, which showed debris in the lateral ventricles consistent with ventriculitis-likely secondary to meningitis. The blood, urine, and cerebrospinal fluid (CSF) cultures collected during our evaluation produced no growth; however, blood cultures from the rural ED eventually grew Salmonella.

A further detailed history revealed that the infant and her parents had been living with a family friend who owned an iguana. According to reports, the iguana had free run of the home and often crawled around and across the infant while she was lying on a blanket on the floor. The patient's parents were not aware of the diseases associated with reptile contact. Due to concerns over the social situation, the patient was kept in the hospital for the entire recommended 21-day course of antibiotic therapy, during which time the parents received assistance finding alternate living arrangements.

\section{Discussion}

\section{Current Practice Guidelines for Managing} Febrile Infants

Current guidelines from the American Academy of Pediatrics (AAP) and the American College of Emergency Physi- cians (ACEP) recommend a full sepsis work-up for all neonates (ie, ages 0 to 28 days) who present with a fever (defined as a rectal temperature $\geq 100.4^{\circ} \mathrm{F}$ ). ${ }^{1,2}$ The probability of a serious bacterial infection (SBI) in patients in this age group who present with fever is approximately $12 \% .^{3}$ A full sepsis work-up generally includes a CBC, blood cultures, urinalysis with culture, CSF analysis with culture, and stool cultures if diarrhea is present.

Current guidelines for infants 29 to 90 days of age who present with fever differ between professional associations. The AAP and the American Academy of Family Physicians recommend the following for children in this age range: laboratory evaluation with CBC, blood cultures, CSF analysis, urinalysis, and culture. If laboratory evaluation reveals a WBC of less than $15 \times 10^{9} / \mathrm{L}$ with an absolute neutrophil count of less than $10 \times 10^{9} / \mathrm{L}$, along with a normal CSF and urinalysis, the patient can be given IM ceftriaxone and followup arranged in 24 hours. This approach is recommended for patients who are otherwise healthy, nontoxic at presentation, and under the care of a responsible adult. ${ }^{4,5} \mathrm{By}$ comparison, the Philadelphia protocol, though suggesting an identical work-up, recommends against the use of antibiotics in infants deemed at low risk for SBI. ${ }^{6}$

The ACEP does not specifically endorse a management strategy for febrile infants in the 29- to 90-day age group, but instead acknowledges that no age cut-off within this group can be considered absolute when determining management strategy, and suggests that children up to 60 days old should be managed in a manner similar to neonates. ${ }^{2}$ The published guidelines do not include consideration of specific history exposure in the management recommendations.

\section{Typhoidal Serotypes}

Salmonella can be divided into typhoidal (including $S$ typhi and $S$ paratyphi) and nontyphoidal serotypes (NTS), with 


\section{Summary}

Emergency physicians may underestimate the likelihood of SBI in otherwise well-appearing febrile infants. While certain aspects of the history and physical examination in a febrile, well-appearing infant have been shown to correlate with an increased risk of SBI, no single finding can definitively rule in or rule out the disease. ${ }^{20}$ Opinions differ as to optimal management strategies for febrile, well-appearing infants outside the neonatal period. However, an appropriate level of clinical suspicion, within the context of a thorough investigation into the infant's health history and social situation, can aid the clinician and guide treatment and disposition.

\section{References}

1. Baraff LJ, Bass JW, Fleisher GR, et al. Practice guideline for the management of infants and children 0 to 36 months of age with fever without source. Agency for Health Care Policy and Research. Ann Emerg Med. 1993;22(7):1198-1210. Erratum in Ann Emerg Med. 1993;22(9):1490.

2. American College of Emergency Physicians Clinical Policies Committee; American College of Emergency Physicians Clinical Policies Subcommittee on Pediatric Fever. Clinical policy for children younger than three years presenting to the emergency department with fever. Ann Emerg Med. 2003;42(4):530-545.

3. Kadish HA, Loveridge B, Tobey J, Bolte RG, Corneli HM. Applying outpatient protocols in febrile infants 1-28 days of age: can the threshold be lowered? Clin Pediatr (Phila). 2000;39(2):81-88.

4. Sur DK, Bukont EL. Evaluating fever of unidentifiable source in young children. Am Fam Physician. 2007;75(12):1805-1811.

5. Baraff LJ, Lee SI, Schriger DL. Outcomes of bacterial meningitis in children: a meta-analysis. Pediatr Infect Dis J. 1993;12(5):389-394.

6. Baker MD, Bell LM, Avner JR. Outpatient management without antibiotics of fever in selected infants. N Eng J Med. 1993;329(20):1437-1441.

7. Gal-Mor O, Boyle EC, Grassl GA. Same species, different diseases: how and why typhoidal and nontyphoidal Salmonella enterica serovars differ. Front in Microbiol. 2014;5:391.

8. Stuart BM, Pullen RL. Typhoid: clinical analysis of 360 cases. Arch Intern Med (Chic). 1946;78(6): 629-661.

9. Olsen SJ, Bleasdale SC, Magnano AR, et al. Outbreaks of typhoid fever in the United States, 1960-99. Epidemiol Infect. 2003;130(1):13-21.

10. Newton AE, Routh JA, Mahon BE. Typhoid and Paratyphoid Fever. In: Brunette GW, Kozarsky PE, Cohen NJ, et al, eds. CDC Health Information for International Travel. New York, NY: Oxford University Press; 2016. http://wwwnc.cdc.gov/ travel/yellowbook/2016/infectious-diseases-relatedto-travel/typhoid-paratyphoid-fever. Updated July 10, 2015. Accessed June 13, 2016.

11. McGovern VJ, Slavutin LJ. Pathology of Salmonella colitis. Am J Surg Pathol. 1979;3(6):483-490.

12. Liu SL, Ezaki T, Miura H, Matsui K, Yabuuchi E. Intact motility as a Salmonella typhi invasion-related factor. Infect Immun. 1988;56(8):1967-1973.

13. House D, Wain J, Ho VA, et al. Serology of typhoid fever in an area of endemicity and its relevance to diagnosis. J Clin Microbiol. 2001;39(3):1002-1007.

14. Hoelzer K, Moreno Switt AI, Wiedmann M. Animal contact as a source of human non-typhoidal salmonellosis. Vet Res. 2011;42(1):34.

15. Murphy D, Oshin F. Reptile-associated salmonellosis in children aged under 5 years in South West England. Arch Dis Child. 2015;100(4):364-365.

16. Iwamoto M. Infectious diseases related to travel. In: Brunette GW, Kozarsky PE, Cohen NJ, et al, eds. CDC Health Information for International Travel. New York, NY: Oxford University Press; 2016. http://wwwnc.cdc.gov/travel/yellowbook/2016/infectious-diseases-related-to-travel/salmonellosis-nontyphoidal. Updated July 10, 2015. Accessed June 13, 2016.

17. Meyer Sauteur PM, Relly C, Hug M, Wittenbrink MM, Berger C. Risk factors for invasive reptile-associated salmonellosis in children. Vector-Borne and Zoonotic Dis. 2013;13(6):419-421.

18. Gasem MH, Keuter M, Dolmans WM, Van Der Ven-Jongekrijg J, Djokomoeljanto R, Van Der Meer JW. Persistence of Salmonellae in blood and bone marrow: randomized controlled trial comparing ciprofloxacin and chloramphenicol treatments against enteric fever. Antimicrob Agents Chemother. 2003;47(5):1727-1731.

19. Price EH, de Louvois J, Workman MR. Antibiotics for Salmonella meningitis in children. J Antimicrob Chemother. 2000;46(5) 653-655.

20. Craig JC, Williams GJ, Jones M, et al. The accuracy of clinical symptoms and signs for the diagnosis of serious bacterial infection in young febrile children prospective cohort study of 15781 febrile illnesses. BMJ. 2010;340:c1594. 